InIFolPharm

Multiple Sklerose

\section{Patienten profitieren multidimensional}

Bei MS-Patienten mit Response auf Fampridin (Fampyra ${ }^{\circledR}$ bessern sich innerhalb der ersten zwei Wochen Gehfähigkeit, kognitives Leistungsvermögen, Stimmung und Tatkraft. Dies ist ein Zwischenergebnis einer noch laufenden Beobachtungsstudie. Die Auswertung basiert auf den Daten von 146 aus 196 in die „Dresdener Studie“ eingeschlossenen MS-Patienten. Der EDSS lag zwischen 4 und 7 (im Mittel 5,4) Punkten. Professor Tjalf Ziemssen, Leiter des MS-Zentrums am Universitätsklinikum Dresden, hob hervor, dass nicht nur die Fampridin-Responder, sondern auch die Nonresponder nachverfolgt werden. Davon erhoffe man sich Informationen zum Verlauf der Behinderung ohne Fampridin-Therapie. Das Responsekriterium (Steigerung der Gehgeschwindigkeit im T25FW-Test um mindestens eine Sekunde nach zweiwöchiger Therapie) erfüllten 71 (49\%) der
Studienteilnehmer. Im Mittel waren sie $25 \%$ schneller als vor Therapiebeginn ( $\mathrm{p} \leq 0,0001)$ mit einer um $23 \%$ verlängerten Gehstrecke im 2-MWT ( $\mathrm{p} \leq 0,0001)$. Die objektive Verbesserung der Mobilität deckte sich in etwa mit der subjektiven Einschätzung der Patienten anhand von MSWS-12 $(p \leq 0,0001)$ und SCGI $(\mathrm{p} \leq 0,0001)$. Als spannend bezeichnete Ziemssen den Befund, dass Fampridin bei den Respondern auch positive Auswirkungen auf Kognition und Konzentration (PASAT) $(\mathrm{p}=0,001)$ sowie Depression (ADS-L) $(\mathrm{p} \leq 0,0001)$ und Fatigue (WEI$\mathrm{MuS})(\mathrm{p} \leq 0,0001)$ hat, was sich auch in einer besseren körperlichen Lebensqualität (FS-36) niederschlug.

Gabriele Blaeser-Kiel, freie Medizinjournalistin

Satellitensymposium „Ziele der MS-Therapie: Illusion oder Realität" beim 85. DGN-Kongress, Hamburg, 27.9.2012. Veranstalter: Biogen Idec

\section{Erste Daten der PRIMA-Studie vorgestellt}

Die Behandlungsoptionen bei CIDP (chronisch inflammatorische demyelinisierende Polyradikuloneuropathie) sind eingeschränkt. Die ICE-Studie zeigte kurz- und mittelfristig einen Nutzen der intravenösen Anwendung von Immunglobulinen (IVIG-Therapie). Sie führte auch zur Zulassung des dort verwendeten Präparates für die CIDP-Therapie, erklärte Professor Claudia Sommer, leitende Oberärztin an der Neurologischen Universitätsklinik Würzburg.

IgPro10 (Privigen ${ }^{\circledR}$ ) ist ein polyvalentes Immunglobulin für die intravenöse Anwendung, zugelassen für die Substitutionstherapie bei primären und sekundären Immundefekten, die Immunmodulationstherapie bei Immunthrombozytopenie, das Guillan-Barré-Syndrom, den Morbus Kawasaki und die allogene Knochenmarktransplantation.

In der PRIMA(The Privigen ${ }^{\circledast}$ Impact on Mobility and Autonomy)-Studie wurde nun das $10 \%$ ige IgPro10 auch in der Indikation CIDP geprüft. In die einarmige offene multizentrische Studie wurden insgesamt 28 Patienten mit CIPD eingeschlossen, von denen 13 bereits mit IVIG vorbehandelt waren. Als Startdosis erhielten sie 2 g/kgKG IgProl0, anschließend wurde über 22 Wochen IgPro10 1g/ kgKG alle drei Wochen appliziert.

Primärer Endpunkt war die Responderrate im INCAT(Inflammatory Neuropathy Cause and Treatment)-Score, definiert als „klinisch bedeutsame Verbesserung“ (Reduktion um mindestens einen Punkt). Diesen Endpunkt erreichten insgesamt 60,7\% der Patienten. IVIG-vorbehandelte Patienten profitierten dabei häufiger $(79,6 \%)$ als neu behandelte Patienten (46,7\%). Die Hälfte der Responder sprach sehr rasch innerhalb der ersten vier Wochen an. IgProl0 konnte bei den CIDP-Patienten die Schwäche vermindern, maximale Griffstärke und Muskelkraft verbessern und den Verlust der motorischen Funktionen verlangsamen. Häufigste Nebenwirkung waren Kopfschmerzen.

Dr. Beate Fessler, freie Medizinjournalistin

Satellitensymposium „Evidenz heute - offene Fragen für morgen: Aktuelle Studien zu Immunglobulinen und ein interessanter Fall", Hamburg, 27.9.2012. Veranstalter: CSL Behring

\section{Alzheimer-Demenz}

Das Unternehmen Actavis hat ab sofort den N-Methyl-D-Aspartat-(NMDA)Rezeptorantagonisten Memantin in das Portfolio aufgenommen. Memantinhydrochlorid Alchemia Actavis ${ }^{\circledR}$ ist laut Hersteller bioäquivalent zu den Erstanbieterprodukten.

Die Filmtabletten sind verfügbar in den Wirkstärken $10 \mathrm{mg}$ (teilbar) und $20 \mathrm{mg}$ sowie in den Zwischenstärken $5 \mathrm{mg}$ und $15 \mathrm{mg}$.

Nach Informationen von Actavis

\section{Schlaganfallprophylaxe}

Die Europäische Kommission hat Apixaban (Eliquis ${ }^{\circledast}$ ) zur Prophylaxe von Schlaganfällen und systemischen Embolien bei erwachsenen Patienten mit nicht-valvulärem Vorhofflimmern und einem oder mehreren Risikofaktoren zugelassen. Es ist weltweit die erste Zulassung für Apixaban zur Schlaganfallprophylaxe bei Patienten mit nicht-valvulärem Vorhofflimmern.

Apixaban gehört als direkter, oraler Faktor-Xa-Inhibitor zu einer neuen therapeutischen Klasse. Apixaban hat sich in der Risikoreduktion von drei wichtigen Endpunkten - Schlaganfall und systemische Embolien, schwere Blutungen und Gesamtmortalität - gegenüber Warfarin als überlegen erwiesen. Die Zulassung von Apixaban basiert auf den beiden Phase-III-Studien ARISTOTLE und AVERROES, in denen insgesamt cirka 24.000 Patienten mit nicht-valvulärem Vorhofflimmern im bislang umfassendsten, abgeschlossenen Studienprogramm zu dieser Patientengruppe untersucht wurden. Das klinische Studienprogramm mit Apixaban ist das einzige Phase-III-Studienprogramm unter den neuen oralen Antikoagulanzien, das die Wirksamkeit und Verträglichkeit von Apixaban gegenüber Aspirin bei Patienten, die für die Therapie mit Vitamin-K-Antagonisten nicht geeignet sind, untersucht hat.

Nach Informationen von Pfizer und Bristol-Myers Squibb 